\title{
An Empirical Investigation of Union-related Factors Contributing to Labour-Management Relationship in Manufacturing Firms in Sri Lanka*"
}

\author{
H.H.D.N.P.Opatha
}

\begin{abstract}
Existing literature reveals a gap in the empirical knowledge in respect of factors that affect Labour-Management Relationship (LMR) at the firm level. The present study, which was part of a comprehensive study of effects of some union-related factors and management-related factors on LMR, empirically evaluated six union-related factors that could have an effect on LMR namely union satisfaction at work, grievance handling, worker discipline administration, worker performance evaluation, union attitudes to management, and competence of union officials. The study involved 202 union officials who were selected from 23 unionised manufacturing firms in Sri Lanka. The results indicated that all the independent variables were positively and significantly correlated to LMR. However, results of regressing the independent variables on LMR showed that union satisfaction at work, grievance handling, worker discipline administration, worker performance evaluation, and union attitudes to management are strong predictors of LMR while competence of union officials is a weak predictor. On the whole, the independent variables accounted for almost $70 \%$ of the variance in the LMR. The paper is of empirical and theoretical importance as the findings confirmed an original explanatory model of LMR that could enhance our understanding of the dynamics of LMR and can be applied to enhance LMR in the manufacturing sector in Sri Lanka.
\end{abstract}

Key words: Grievance Handling, Labour-Management Relationship, Worker Performance Evaluation, Worker Discipline Administration, Union Satisfaction at Work

Acknowledgement: This paper was published in Sasin Journal of Management, Vol 11, $\mathrm{Nu}$ 1, 2005 (the Journal of Sasin Graduate Institute of Business Administration of Chulalongkorn University) and only abstract has been published here. 


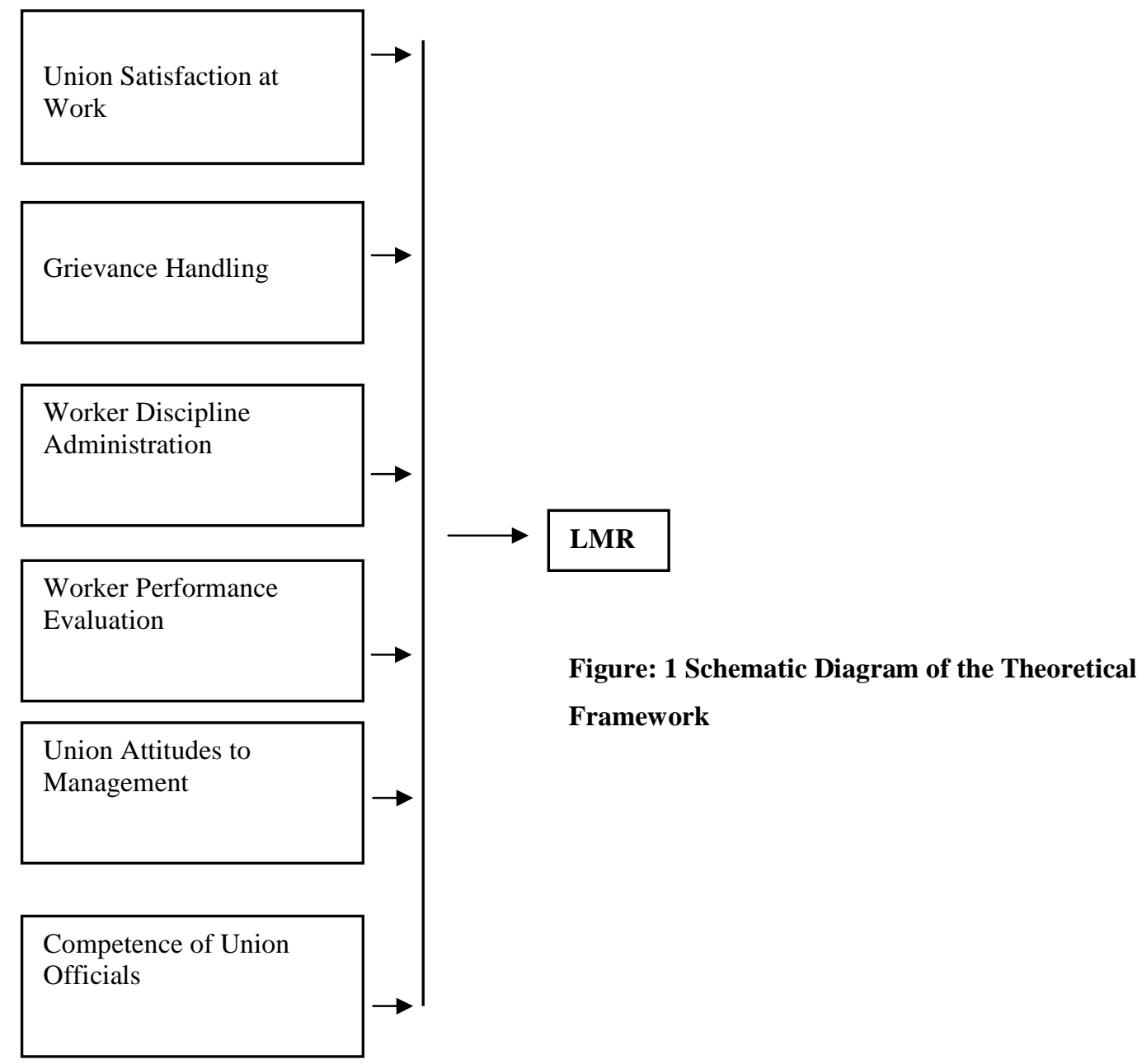

Table: 1 Pearson Correlation Matrix for all Union-related Variables

\begin{tabular}{|l|l|l|l|l|l|l|l|l|}
\hline No & Variables & 1 & 2 & 3 & 4 & 5 & 6 & 7 \\
\hline 1 & LMR & 1.0 & & & & & & \\
\hline 2 & Union Satisfaction at work & $.661^{* *}$ & 1.0 & & & & & \\
\hline 3 & Grievance Handling & $.560^{* *}$ & $.670^{* *}$ & 1.0 & & & & \\
\hline 4 & $\begin{array}{l}\text { Worker Discipline } \\
\text { Administration }\end{array}$ & $.594^{* *}$ & $.425^{* *}$ & $.328^{* *}$ & 1.0 & & & \\
\hline
\end{tabular}


Sri Lankan Journal of Human Resource Management

\begin{tabular}{|l|l|l|l|l|l|l|l|l|}
\hline 5 & $\begin{array}{l}\text { Worker Performance } \\
\text { Evaluation }\end{array}$ & $.309^{* *}$ & .011 & -.037 & -.031 & 1.0 & & \\
\hline 6 & $\begin{array}{l}\text { Union Attitudes to } \\
\text { Management }\end{array}$ & $.594^{* *}$ & $.551^{* *}$ & $.343^{* *}$ & $.249^{* *}$ & $.428^{* *}$ & 1.0 & \\
\hline 7 & $\begin{array}{l}\text { Competence of Union } \\
\text { Officials }\end{array}$ & $.174^{* *}$ & .221 & .079 & .045 & .086 & $.174^{* *}$ & 1.0 \\
\hline
\end{tabular}

$* * \mathrm{p}<.0 .01 \quad \mathrm{n}=202$

Table: 2 Aggregate Impact of the Independent Variables on LMR

\begin{tabular}{|l|l|l|l|l|l|}
\hline $\mathrm{R}$ & R Square & $\begin{array}{l}\text { Adjusted } \\
\text { R Square }\end{array}$ & $\begin{array}{l}\text { Std.Error of } \\
\text { the Estimate }\end{array}$ & F Value & Sig. F \\
\hline .835 & .698 & .688 & 9.0964 & 74.989 & 0.0005 \\
\hline
\end{tabular}

Table: 3 Influence of the Independent Variables on LMR

\begin{tabular}{|l|l|l|l|}
\hline Variable & Std.Beta & $\mathrm{T}$ & Sig. t \\
\hline Union Satisfaction at Work & $\begin{array}{l}.245 \\
(.057) \\
.216\end{array}$ & 3.820 & .000 \\
Wrievance Handling & $\begin{array}{l}(.059) \\
.381\end{array}$ & 8.042 & .000 \\
Worker Discipline Administration & $\begin{array}{l}(.040) \\
.247 \\
(.051) \\
.178 \\
(.055) \\
\text { Worker Performance Evaluation }\end{array}$ & 5.397 & .000 \\
Union Attitudes to Management & $\begin{array}{l}.038 \\
(.055)\end{array}$ & .828 & .000 \\
Competence of Union Officials & .409 \\
\hline
\end{tabular}

(Standard Error in Parentheses)

Table: 4 Stepwise Multiple Regression Analysis: Predictors of LMR

\begin{tabular}{|l|l|l|l|l|}
\hline Variables & $\mathrm{R}^{2}$ & $+\mathrm{R}^{2}$ & Beta & Sig t \\
\hline 1. Union Satisfaction at Work & 0.436 & & .255 & .0005 \\
\hline 2. Worker Discipline Administration & 0.556 & 0.120 & .379 & .0005 \\
\hline 3. Worker Performance Evaluation & 0.656 & 0.100 & .249 & .0005 \\
\hline 4. Grievance Handling & 0.680 & 0.024 & .212 & .0005 \\
\hline 5. Union Attitudes to Management & 0.697 & 0.017 & .179 & .001 \\
\hline R Square: 697 Adjusted R Square: 689 & $\mathrm{F}=89.995$ & \multicolumn{2}{|c|}{ D.F. $=201$}
\end{tabular}

Sig.F $=.0005$ 
Sri Lankan Journal of Human Resource Management 